\title{
Strategic factors in a lexical-decision task: Evidence for automatic and attention-driven processes
}

\author{
KEN den HEYER, KEVIN BRIAND, and GARY L. DANNENBRING \\ St. Francis Xavier University, Antigonish, Nova Scotia
}

\begin{abstract}
The present study examined strategic factors in a semantic-priming, lexical-decision task. The first experiment demonstrated that the greater the proportion of related word-word pairs to unrelated word-word pairs, the greater the amount of facilitation, a result which is consistent with others reported in the literature. The second experiment demonstrated that this strategic factor apparently requires that sufficient time (at least several hundred milliseconds) be available for the processing of the priming word, and thus is probably caused by attention-driven processes. The third experiment replicated and extended the results of the first two studies by demonstrating that prime-target stimulus onset asynchrony is an important limiting factor in determining whether such proportion-induced strategic factors are involved in word-recognition processes, even when no other aspect or variable of the procedure changes. The results are discussed in the context of Posner and Snyder's (1975a) two-process model of word recognition.
\end{abstract}

Recent work in the area of word recognition has generated support for the notion that two different kinds of processes can operate in word identification: namely, processing that can be considered automatic and processing that can be considered intentional, or attention driven (Neely, 1977; Posner \& Snyder, 1975a, 1975b). Some of the support for such a two-process model has come from investigations in which the effect of context on lexical decisions is studied. In such studies (e.g., Meyer \& Schvaneveldt, 1971), subjects typically must decide whether a letter string is a word or a nonword. The usual finding is that "yes" decisions are facilitated if the target item is preceded by a related word (e.g., doctor-nurse) as compared with an unrelated word (e.g., lamp-nurse). A common explanation for this facilitation, or priming, effect is that the processing of the first item, or prime, activates representations of semantically related items, which in turn facilitates the encoding of a second word if it is related to the prime (Collins \& Loftus, 1975; Posner \& Snyder, 1975a). Although some researchers have argued that priming can be better accounted for by mechanisms other than spreading activation (Becker, 1979, 1980; Schvaneveldt \& McDonald, 1981), considerable support exists in the literature for the spreading-activation notion, especially in the context of Posner and Snyder's (1975a)

This research was supported by grants from the Natural Science and Engineering Research Council of Canada and the St. Francis Xavier University Council for Research to G. Dannenbring and K. den Heyer. Reprint requests may be sent to Ken den Heyer, Department of Psychology, St. Francis Xavier University, Antigonish, Nova Scotia B2G 1CO, Canada. two-process model (Fischler, 1977; Fischler \& Goodman, 1978; Foss, Cirilo, \& Blank, 1979; Neely, 1976, 1977; Neill, 1979; Stanovich \& West, 1979, 1981).

A recent trend in this research has been to investigate the possible role of strategic factors in performing these tasks (see Coltheart, 1978, for a general review of research on strategic factors in lexical access). A study by Tweedy, Lapinski, and Schvaneveldt (1977) suggested that strategic components play a large role in semantic priming. These investigators found that the amount of priming depended on the proportion of related pairs in a lexicaldecision task. The number of related items, as a proportion of word-word pairs, correlated positively with the amount of facilitation. Thus, subjects were somehow able to take advantage of the fact that many word-word pairs were related in order to facilitate their lexical decisions. Subsequently, Tweedy and Lapinski (1981) were able to demonstrate that this strategic component could vary within a testing session. The process of a subject's implementing a strategic component was clearly demonstrated, because the amount of facilitation depended on the tendency for a subject to see more or fewer related pairs of items as the testing session proceeded.

Together, the studies by Tweedy and Lapinski (1981) and Tweedy, Lapinski, and Schvaneveldt (1977) argue for the importance of a strategic component in semantic priming. However, neither report actually specifies whether this strategic factor is an automatic or an attention-driven process. Presumably, attention-driven facilitation requires considerably more processing time than does automatic 
spreading activation (Neely, 1977; Posner \& Snyder, 1975a); thus, if only a brief time elapses between prime and target items, attention-driven processes do not have sufficient time to occur, and the effects of automatic processes alone should be observed. Neely (1977) demonstrated that at prime-target stimulus onset asynchronies (SOAs) of greater than $250 \mathrm{msec}$, contextual facilitation occurred when the prime was related to the target, and inhibition occurred when there was an unexpected shift in the prime-target relationship. Inhibitory effects, however, did not occur at SOAs as short as $250 \mathrm{msec}$, whereas facilitation can be demonstrated even when the interval between prime and target is as short as $40 \mathrm{msec}$ (Fischler \& Goodman, 1978). All of these results are consistent with the two-process theory of Posner and Snyder (1975a).

The studies by Tweedy and Lapinski (1981) and Tweedy, Lapinski, and Schvaneveldt (1977) used a procedure in which the subjects were required to make two lexical decisions per trial: one for the prime and one for the target. The target was not presented until $100 \mathrm{msec}$ after completion of the response to the prime. It is apparent that both fast, automatic and slow, attention-driven factors were free to operate in such a procedure, since the time duration between the presentation of the letter-string pairs was at least $500 \mathrm{msec}$, and probably much more. One might ask whether the strategic component hypothesized by Tweedy and Lapinski (1981) could still be effective if very short interstimulus intervals were used. Finding it to be ineffective would demonstrate another difference between automatic and attention-driven processes and would provide additional support for a two-factor theory of word recognition.

The present research first attempted to replicate the results of Tweedy et al. (1977) in order to establish that such a strategic factor is a reliable phenomenon when different stimuli, or at least independently chosen stimuli, and different subjects are used (see Clark, 1973, and Wike \& Church, 1976). Two subsequent experiments then examined the effect of limiting controlled, attention-driven factors by using a short SOA between prime and target.

\section{EXPERIMENT 1}

\footnotetext{
Method

Subjects. Forty-eight students were recruited from St. Francis Xavier University. The subjects were paid for their participation in the experiment.

Apparatus. Sixty-four pairs of related words were selected from norms supplied by Battig and Montague (1969) and Palermo and Jenkins (1964). These 64 pairs were so arranged as to create 56 related pairs and 8 unrelated pairs for the high-proportion $(\mathrm{H})$ condition, 32 related and 32 unrelated pairs for the mediumproportion (M) condition, and 8 related pairs and 56 unrelated pairs for the low-proportion (L) condition. These three different sets were arbitrarily created by re-pairing associative pairs, with different pairings occurring for each subject. In addition, 64 other
}

words were selected from the norms mentioned above. These were used as targets and primes in the 32 word-nonword and 32 nonwordword pairs. A further 96 words were selected from the norms to create nonwords by randomly changing one letter in each syllable. These were used in 80 pairs of letter strings containing 32 nonwordword pairs, 32 word-nonword pairs, and 16 nonword-nonword pairs. Thus, a complete stimulus set consisted of 64 word-word pairs, 32 nonword-word pairs, 32 word-nonword pairs, and 16 nonword-nonword pairs, for a total of 144 pairs of items. The three different stimulus sets used differed only with respect to the proportion of related word-word pairs. The apparatus for stimulus presentation consisted of the Radio Shack TRS-80 Model I microcomputer system. Only uppercase letters were used in the stimuli.

Procedure. In accord with the procedure used by Tweedy et al. (1977), the three different stimulus sets were used with three different groups of subjects (16 subjects in each group). Each trial began with a 200 -msec warning signal consisting of a row of asterisks, which was then replaced by the prime. The subject then made a lexical decision in response to the prime. When the response was made, the prime was replaced by a row of asterisks for $500 \mathrm{msec}$; the target was then presented immediately below the row of asterisks. After response (lexical decision) to the target, the screen was cleared, the next trial number was displayed in the upper left-hand corner of the screen, and after approximately $500 \mathrm{msec}$, the next trial began.

All 144 pairs of items were administered in a random sequence, and each subject received a unique random sequence. If a subject made an error on either the prime or the target, the pair of items was returned to the yet-to-be presented items. Thus, after completion of the experimental session, each subject had responded correctly to all 288 items in the 144 pairs.

All subjects received a minimum of 15 practice trials. The subjects received no information with respect to the proportion of related-pairs manipulation or even to the fact that they would see related pairs. Responses were made by pressing one of two keys on the TRS-80 keyboard. Each subject used his or her preferred hand for the "yes, it is a word" decision.

\section{Results}

The standard deviation of reaction times (RTs) to word targets was calculated for each subject. RTs to the targets in the word-word conditions that were more than 1.96 standard deviations away from the overall mean RT to word targets (for the subject) were deleted. Mean RTs, percent errors to targets, and percent of trials deleted as too extreme are given in Table 1. Mean RTs were calculated for the related and unrelated word-word conditions for each subject and are shown in Figure 1. These means were then analyzed by a 3 (stimulus set or proportion) $\times 2$ (related-unrelated) analysis of variance. Significant effects were obtained for the related-unrelated factor $[F(1,45)=54.42, p<.001]$ and the stimulus set $x$ related-unrelated interaction $[\mathrm{F}(2,45)=7.32, \mathrm{p}<$ $.01]$. As can be seen in Figure 1, responses for related targets were faster than for unrelated targets (the basic priming effect), and the amount of priming decreased as the proportion of related pairs decreased. Error rates for word targets were low (less than 3\%) and gave no indication of being related to any variable of interest (see Table 1).

A linear-regression equation for the average amount of facilitation for each of the three stimulus sets was calculated (Tweedy et al., 1977). The equa- 
Table 1

Mean Reaction Times (in Milliseconds), Percent Errors, and Percent Thrown Out in Experiment 1 Conditions

\begin{tabular}{|c|c|c|c|c|c|c|c|c|c|c|c|c|c|c|c|}
\hline \multirow[b]{2}{*}{ Proportion } & \multicolumn{3}{|c|}{ W-WR } & \multicolumn{3}{|c|}{ W-WU } & \multicolumn{3}{|c|}{ NW-W } & \multicolumn{3}{|c|}{ W-NW } & \multicolumn{3}{|c|}{ NW-NW } \\
\hline & RT & $\mathrm{PE}$ & PTO & $\mathrm{RT}$ & PE & PTO & RT & $\mathrm{PE}$ & PTO & RT & $\mathrm{PE}$ & PTO & RT & PE & PTO \\
\hline Low & 525 & 1.92 & 3.91 & 553 & 1.92 & 5.02 & 616 & 2.34 & 5.66 & 848 & 9.77 & $\mathcal{J}$ & 810 & 3.91 & 5.86 \\
\hline Medium & 535 & 1.42 & 3.32 & 559 & 1.70 & 5. & 604 & 3.13 & & 772 & 23.44 & 4.10 & 728 & 13.54 & 7.42 \\
\hline High & 499 & 0.96 & 3.01 & 569 & 6.73 & 7.81 & 579 & 2.68 & 6.64 & 709 & 4.46 & 3.71 & 705 & 6.25 & 7.81 \\
\hline
\end{tabular}

Note $-W-W R=$ word-word, related $; W-W U=$ word-word, unrelated $; N W-W=$ nonword-word $; W-N W=$ word-nonword $; N W-N W=n o n-$ word-nonword; $P E=$ percent error; $P T O=$ percent thrown out.

tion is: facilitation (in $\mathrm{msec}$ ) $=13+56.00 \mathrm{p}$, where $\mathrm{p}$ is the proportion of related items in the three stimulus sets $(\mathrm{p}=.125, .5$, and .875 for the $\mathrm{L}, \mathrm{M}$, and $\mathrm{H}$ stimulus sets, respectively). Although the intercept differs from that reported by Tweedy et al. (1977) $(26 \mathrm{msec})$, the slopes are almost identical (56 and 57.36, respectively).

Further regression equations were calculated for mean RTs for the related and unrelated word-word conditions as a function of proportion in the three stimulus sets. These functions are: RT (related) (in $\mathrm{msec}$ ) $=537-34.67 \mathrm{p}$ and RT (unrelated) (in msec) $=550+21.33 \mathrm{p}$.

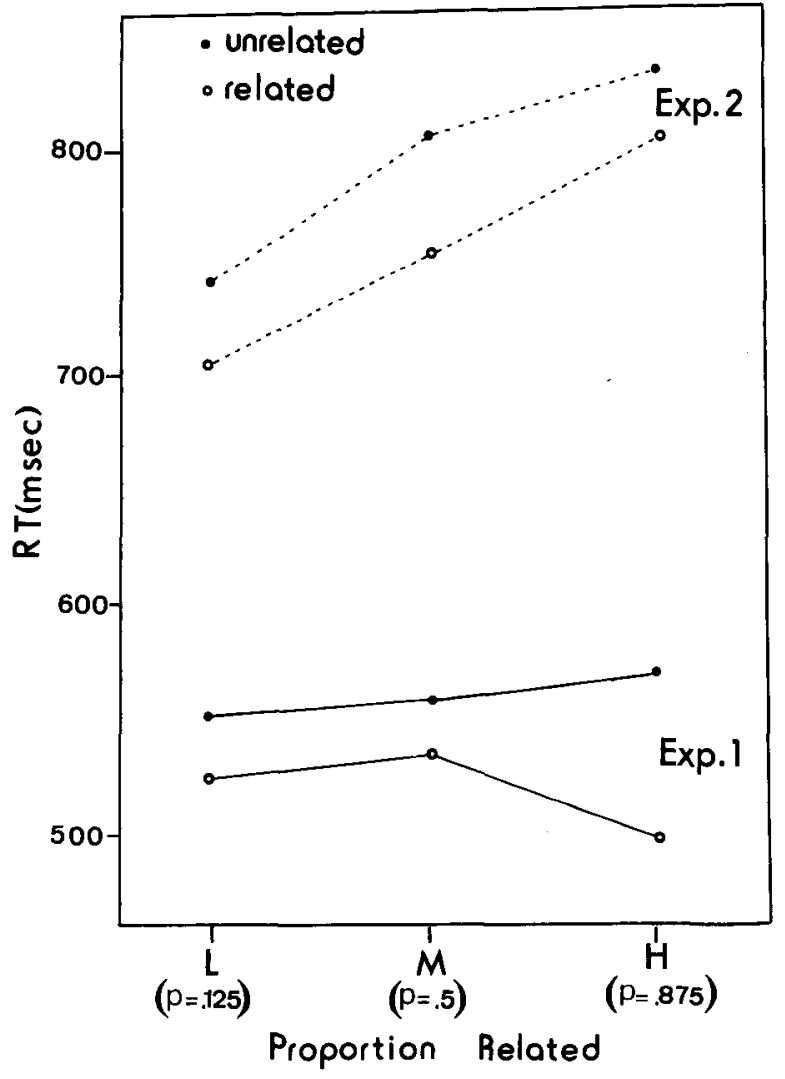

Figure 1. Mean RTs for related and unrelated words in Experiments 1 and 2 as a function of the proportion of related wordword pairs.
Again, these results are similar to those of Tweedy et al. (1977). The functions they obtained were: RT (related) (in msec) $=489-45.36 \mathrm{p}$ and RT (unrelated) (in $\mathrm{msec}$ ) $=515+12.00 \mathrm{p}$.

\section{Discussion}

The results of the present experiment demonstrate a clear relationship between the proportion of relateditems pairs and the amount of facilitation. As Tweedy et al. (1977) suggested, "Contextual effects are at least partially produced by the observer's increased ability to take advantage of the predictability of the stimulus sequence when it contains frequent instances of semantically related word pairs" (p. 88).

The question we next wished to address was whether this strategic factor operates quickly in a priming trial or whether it operates more slowly, in line with an attention-driven process, such as postulated by Posner and Snyder (1975a). Experiment 2 addressed this question by limiting the amount of processing time for the prime.

\section{EXPERIMENT 2}

\begin{abstract}
Method
Subjects. Forty-eight students were recruited from St. Francis Xavier University. The subjects were paid for their participation. None of the subjects in this experiment had participated in the previous experiment.

Procedure. The apparatus, stimuli, and procedure were identical to those of Experiment 1, with the following exceptions. First, the subjects made only one lexical decision (this, to the target) per trial. Second, the prime was presented, and $75 \mathrm{msec}$ later the target was presented below the prime, which remained in view. The subjects were instructed to respond to the target as quickly as possible. Third, on random occasions, but, on the average, on every 10th trial, the subjects were asked if the prime was or was not a word. It should also be noted that because both the prime and the target were cleared from the screen after the subject's decision about the target had been made, the prime was no longer in view when this random check was made.
\end{abstract}

\section{Results}

Mean RTs were calculated as described in Experiment 1 , and extreme trials were deleted (see Table 2). Once again the means for each subject for the wordword trials were analyzed by a 3 (stimulus set) $\times 2$ (related-unrelated) analysis of variance. These results 
Table 2

Mean Reaction Times (in Milliseconds), Percent Errors, and Percent Thrown Out in Experiment 2 Conditions

\begin{tabular}{|c|c|c|c|c|c|c|c|c|c|c|c|c|c|c|c|}
\hline \multirow[b]{2}{*}{ Proportion } & \multicolumn{3}{|c|}{ W-WR } & \multicolumn{3}{|c|}{ W-WU } & \multicolumn{3}{|c|}{ NW-W } & \multicolumn{3}{|c|}{ W-NW } & \multicolumn{3}{|c|}{ NW-NW } \\
\hline & RT & PE & PTO & RT & $\mathrm{PE}$ & PTO & $\mathrm{RT}$ & $\mathrm{PE}$ & PTO & $\mathrm{RT}$ & $\mathrm{PE}$ & PTO & RT & PE & PTO \\
\hline Low & 706 & 0.00 & 6.25 & 742 & 3.08 & 5.13 & 801 & 3.13 & 5.47 & 955 & 18.36 & 5.27 & 895 & 16.41 & 5.86 \\
\hline Medium & 754 & 2.84 & 2.34 & 805 & 4.55 & 4.49 & 856 & 2.08 & 6.44 & 1096 & 5.73 & 5.08 & 1111 & 7.29 & 4.69 \\
\hline High & 804 & 1.79 & 2.57 & 834 & 6.25 & 6.25 & 921 & 3.13 & 7.42 & 1152 & 15.23 & 4.88 & 1121 & 5.47 & 5.08 \\
\hline
\end{tabular}

Note $-W-W R=$ word - word, related $; W-W U=$ word - word, unrelated $; W-W=$ nonword - word $; W-N W=$ word - nonword $; N W-N W=$ nonword-nonword $; P E=$ percent error $; P T O=$ percent thrown out. Accuracy in prime identification: Low $=14.8 ;$ Medium $=7.28$; High $=16.22$.

can be seen in Figure 1. Only the related-unrelated variable proved to be significant $[F(1,45)=14.69$, $\mathrm{p}<.01]$. There was no evidence of any effect due to stimulus set or proportion. Although this latter trend appears obvious in Figure 1, it was in fact far from significant $[F(2,45)<1]$. The critical interaction between proportion and relatedness was also far from significant $[F(2,45)=0.399]$. Additionally, percent error and trials deleted were similarly analyzed. Both the percent error and the percent trials deleted were larger for the unrelated word-word condition than for the related word-word condition $[F(1,45)=6.10$, $\mathrm{p}<.05$, and $\mathrm{F}(1,45)=5.70, \mathrm{p}<.05$, respectively]. More importantly, there was no interaction between proportion and prime type for either the error or the trials-deleted data $[\mathrm{F}(2,5)=0.26$ and $\mathrm{F}(2,45)=1.02$, respectively].

The RT data of Experiment 2 were combined with those of Experiment 1 in a three-factor analysis of variance in which the factors were experiment or task, stimulus set, and related-unrelated word pairs. Main effects of experiment $[F(1,90)=44.77, p<.001]$ and related-unrelatedness $[\mathrm{F}(1,90)=47.48, \mathrm{p}<.001]$ proved to be significant. The three-way interaction was also significant $[F(2,90)=3.13, p<.05]$, again indicating the difference between the results of Experiments 1 and 2 .

\section{Discussion}

Unlike those of Experiment 1, the results of Experiment 2 failed to demonstrate any effect of proportion of related items within a stimulus set on amount of priming. Despite problems in asserting the null hypothesis, it appears likely that the strategic factor the subjects used in Experiment 1 did not operate, or at least operated ineffectively, in Experiment 2.

There are two possible explanations for why this effect of proportion failed to interact with semantic priming in Experiment 2. Because the prime duration preceding target presentation was short and because the subjects did not have to respond to the prime (at least on $90 \%$ of the trials), the subjects may not have been sensitive to the proportion of related pairs manipulation. In other words, since there was both less opportunity and less need to process the prime, the subjects may not have become aware, either explicitly or implicitly, that there was, or was not, a preponderance of related word-word pairs. Thus, one might not expect an interaction between the proportion manipulation and facilitation. Two factors argue against this interpretation. One would expect less priming in Experiment 2 than in Experiment 1 if the prime was not processed or its processing extent diminished. Although the average amount of priming was slightly less in Experiment 2 (35 msec vs. $40.7 \mathrm{msec}$ ), the difference was not significant. A second fact that argues against the diminishedprime-processing hypothesis is the relatively low error rate in Experiment 2 when the subjects were asked whether or not the prime was a word $(12.8 \%)$. Obviously, then, the subjects did process the prime.

The second possible explanation of why the proportion manipulation had no effect stems not from the fact that the subjects did not "notice" the proportion of related pairs, but, rather, from the possibility that the strategic factor induced by a preponderance of related word-word pairs did not have time to operate within the time constraints of the trial presentation. That is to say, the strategic factor operating in Experiment 1 was initiated by presentation of the prime and then operated relatively slowly in its effect upon related semantic representations in memory. Such a possible explanation stems directly from the dual-process model of Posner and Snyder (1975a). In the processing of information such as words, letters, or letter strings, both automatic and strategic processes are operative. The automatic processes are said to be fast acting, whereas the strategic processes operate more slowly and require attention and processing capacity. In the Posner and Snyder (1975a) model, the slow-acting, attention-driven processes incur both benefits and costs in terms of processing efficiency. In Experiment 1, the processing of targets of related pairs in the high-proportion stimulus sets enjoyed an advantage because of the strategic factor induced by the high proportion of related word-word pairs. According to the Posner and Snyder (1975a) model, then, there should have been a corresponding cost for unrelated word-word pairs, al- 
though such costs need not have been symmetrical. As Tweedy et al. (1977) pointed out in their discussion, the benefits in the word-word semantic-priming procedure accrue to a relatively small set of items related to the prime, but the cost may be spread over a relatively large number of unrelated words (all remaining words in the lexicon). In our particular results in Experiment 1, the effect of proportion on the amount of facilitation was a joint function of a decrease in RT for the related items as a function of increasing proportion and of an increase in RT for the unrelated items as a function of increasing proportion of related items, although the negative slope was larger than the positive slope $(-34.67 p$ as compared with $21.33 \mathrm{p}$ ). Thus, it appears that a cost/ benefit kind of strategic factor was operating in the results of Experiment 1, which lends additional support to the interpretation that the lack of a proportion effect in Experiment 2 was due to the fact that the strategic factor is relatively slow in its effects and requires processing capacity.

One difference between the results of Experiment 1 and those of Experiment 2 that deserves some comment is the substantially longer RTs in Experiment 2, as can readily be seen in Figure 1. It is possible that the procedure in the word-word trials in Experiment $1-$ two consecutive responses with the same hand-may have set up some form of response bias that made all responses to word targets faster. If such was the case, then an analysis involving the task variable and a compatibility variable (nonword-word and word-word pairs) should have yielded a significant interaction. Such was not the case $[F(1,90)=$ $1.77, p>.05]$. A more likely explanation for the longer RTs in Experiment 2 is that the short SOA in Experiment 2 delayed the processing of the target until prime processing had reached some stage of completion.

The first two experiments, then, corroborated the finding that increasing the proportion of semantically related word-word pairs facilitates priming. Furthermore, such a strategic factor appears to operate in a relatively slow manner and requires processing capacity. These conclusions, however, are based on a comparison across experiments that employed two different procedures. Their validity would be strengthened if similar results could be obtained in one experiment using identical procedures over different prime-to-target intervals. Experiment 3 was planned accordingly. Additionally, the procedure in Experiment 3 included a "neutral" priming condition. This allowed for a more valid examination of the processing-capacity aspect of the strategic factor. The disadvantage of including a neutral priming condition was that it reduced the overall density of related word-word trials.

\section{EXPERIMENT 3}

\section{Method}

Subjects. One hundred and two students were recruited from St. Francis Xavier University. All subjects were volunteers, and none had participated in the first two experiments.

Apparatus. The same items used in Experiment 2 were included in the present procedure. However, an additional 32 related wordword pairs were taken from a set of synonymity norms (Whitten, Suter, \& Frank, 1979). Sixteen additional nonwords were selected and created as described in Experiment 1 . These nonwords were then paired with neutral primes. The neutral prime consisted of five Xs. Therefore, there were 96 pairs of related words. Thirtytwo of these pairs were chosen randomly and their primes replaced by neutral primes. The remaining 64 pairs of related word-word pairs were then divided into related and unrelated sets in order to achieve high, medium, and low proportions of related pairs, as in the previous experiments. The total stimulus set consisted of 192 pairs of letter strings: 64 of these were word-word pairs, 32 were neutral-prime/word pairs, 32 were nonword-word pairs, 32 were word-nonword pairs, 16 were neutral-prime/nonword pairs, and 16 were nonword-nonword pairs.

Procedure. The procedure was essentially the same as that used in Experiment 2, except that two different prime-target SOAs (75 and 1,000 msec) were used. The two SOA conditions were combined with the three proportion conditions to produce six different experimental conditions, and six groups, of 17 subjects each, were tested.

\section{Results}

Mean RTs were calculated as described in Experiment 1, and the word-word RTs can be seen in Figure 2. Extreme trials were deleted, and the mean RTs, percent errors, and percent deleted are given in Table 3. Word-word RTs were analyzed as in Experiments 1 and 2. Separate analyses were first performed on the 75 - and the 1,000 -msec conditions. In the 75 -msec condition, there was a significant effect only of relatedness $[F(1,48)=17.18, p<.01]$. The proportion effect was not significant $(F<1)$, and the relatedness $\times$ proportion interaction was not significant $[\mathrm{F}(2,48)=2.41, \mathrm{p}>.10]$. In the 1,000 msec condition, there was an overall effect of relatedness $[F(1,48)=30.50, p<.001]$, and the proportion effect was not significant $(F<1)$. The proportion $x$ relatedness interaction, however, was significant $[\mathrm{F}(2,48)=4.53, \mathrm{p}<.025]$.

Regression equations were then calculated for the 1,000-msec condition, as in Experiment 1. First, regression equations for RT in the related and unrelated conditions as a function of proportion were calculated. The solutions are as follows: RT (related) (in msec) $=637-45.33 \mathrm{p}$ and RT (unrelated) (in msec) $=645+21.33 \mathrm{p}$. These are comparable with the analogous equations obtained in Experiment 1. Additionally, an attempt was made to determine trends in costs/benefits as a function of proportion, and the solutions arrived at are as follows: benefits (neutral - related) (in msec) $=15+30.67 \mathrm{p}$; costs (unrelated - neutral) (in msec) $=-7+36.00$ p. Thus, it can 


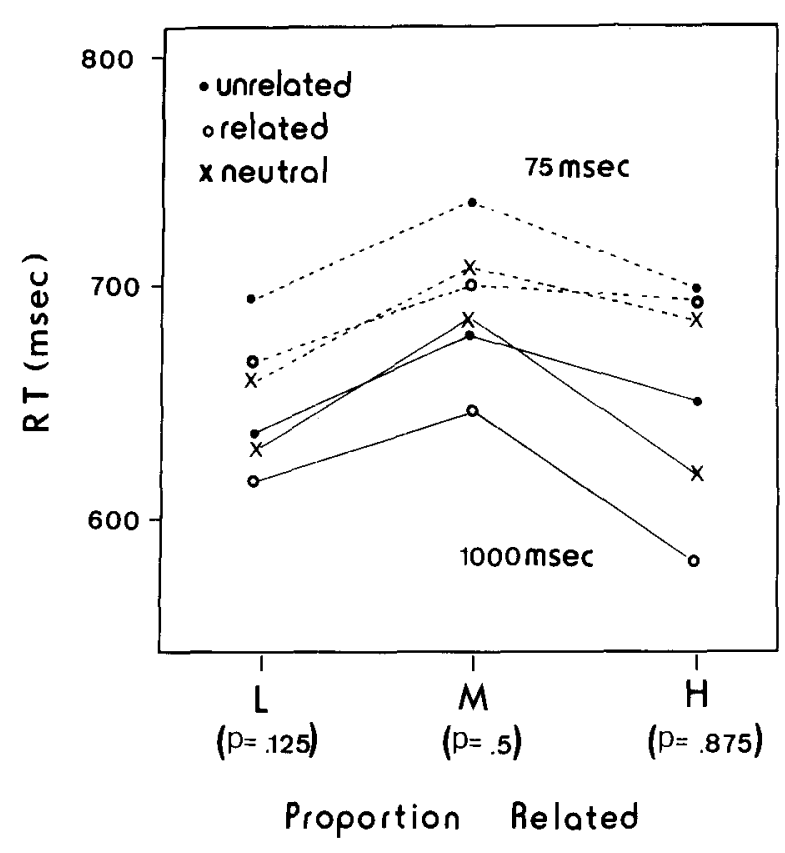

Figure 2. Mean RTs for word targets following related, unrelated, or neutral primes in Experiment 3 as a function of the proportion of related word-word pairs and prime-target SOA.

be seen that for the 1,000-msec condition, both costs and benefits increased as the proportion of related word-word pairs increased.

A three-way analysis of variance with prime-target SOA and proportion as the between-subjects variables and with relatedness as a repeated variable was then carried out. The results were essentially the same as those of the combined analysis of Experiments 1 and 2 . The three-way interaction between relatedness, proportion, and SOA was significant $[F(2,96)$ $=6.48, \mathrm{p}<.005$ ]. Relatedness was also significant $[F(1,96)=48.30, p<.001]$. The SOA $\times$ relatedness interaction was significant $[F(1,96)=3.60, p<.05]$, and the SOA effect was marginal but nonsignificant $[F(1,96)=3.49, \mathrm{p}<.10]$.

The previous analyses were based on only the related and unrelated word-word RTs. Other analyses that included the neutral-prime/word RTs as a third variable on the repeated factors measure were carried out. All these analyses turned up results similar to those obtained when the neutral-prime trials were excluded. For the $75-\mathrm{msec}$ condition, only prime type (related/unrelated/neutral) was significant $[F(2,96)$ $=9.40, \mathrm{p}<.01]$. In the 1,000 -msec condition, both prime type $[F(2,96)=19.15, \mathrm{p}<.01]$ and the prime type $\times$ proportion interaction were significant $[F(4,96)$ $=2.89, \mathrm{p}<.05]$. Finally, an overall three-factor analysis was performed. There was a significant effect of prime type $[F(2,192)=22.53, p<.01]$ and a prime type $\times$ SOA interaction $[F(2,96)=6.09, p<$ $.01]$. Furthermore, the three-way interaction between prime type, proportion, and SOA was significant $[F(4,192)=3.06, p<.05]$. The effect of SOA was marginal, but nonsignificant $[F(1,96)=2.84, p<.10]$.

Analysis of error data, as well as of the number of trials trimmed, revealed no significant effects. Of particular interest was the $75-\mathrm{msec}$ condition, since the lack of an interaction between relatedness and proportion for the RT data may have been masked by either error rates or number of trials trimmed, or both. The appropriate analysis for both the error rates and the number of trials deleted revealed no significant effects due to relatedness, proportion, or the interaction between proportion and relatedness $[F(2,48)=1.64, p>.20$, and $F(2,48)=0.67$ for error and trial deletion $\times$ proportion interactions, respectively].

\section{Discussion}

In general, the results of Experiment 3 corroborated the findings of the first two experiments. That is to

Table 3

Mean Reaction Times (in Milliseconds), Percent Errors, and Percent Thrown Out in Experiment 3 Conditions

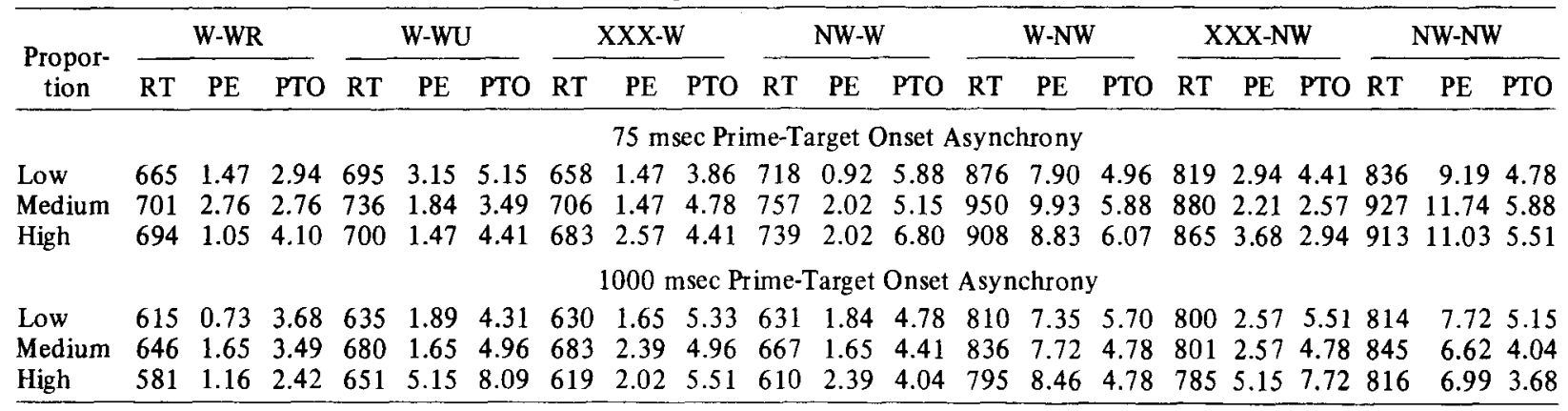

Note $-W-W R=$ word $-w o r d$, related $; \cdot W U=$ word-word, unrelated $; X X X-W=$ neutral prime-word $; W-W=$ nonword-word; $W-N W=$ word-nonword; $X X X-N W=$ neutral prime-nonword; $N W \cdot N W=$ nonword-nonword $; P E=$ percent error; PTO = percent thrown out. Percent error in prime identification: 75-msec condition, Low $=19.15 ;$ Medium $=9.06 ;$ High $=12.84$. 1000-msec condition, Low $=$ 14.15; Medium $=14.33 ;$ High $=17.02$. 
say, the density of related word-word pairs related positively with the magnitude of priming under conditions in which attention-driven processes had an opportunity to operate, which in this case was the 1,000-msec prime-target SOA condition. It should be noted that this finding occurred despite a reduction in related word-word trials density in the overall trial array, a reduction caused by the inclusion of neutralprime/word and neutral-prime/nonword pairs (an extra 48 trials).

Two aspects of the neutral-word trials should be noted. First, with respect to the 75-msec prime-target SOA condition, it seems that neutral-word trials represent a poor benchmark for measuring priming effects. It appears that when the SOA is that brief, the processing of the target is delayed until a certain minimum processing of the prime has been completed. This caused the longer RTs in Experiment 2 than in Experiment 1 and, similarly, in the $75-\mathrm{msec}$ condition than in the $1,000-\mathrm{msec}$ condition in Experiment 3 . Similar results have been reported by Lorch (1982) and Schmidt (1976). The fact that the target RT of the neutral-word trials in the $75-\mathrm{msec}$ condition falls between that of the related and unrelated word-word trials does not indicate that both facilitation and inhibition effects are at work. Rather, whatever minimum compulsory processing of the prime that does take place is more time consuming when the prime is a word rather than a row of Xs. However, and this represents the second aspect of the neutral-prime/word condition, this is not the case with the 1,000 -msec prime-target SOA condition. Here, a neutral-prime condition can act as a suitable baseline for measuring priming effects. A full $1 \mathrm{sec}$ between presentation of the prime and the target allows ample time for the prime to be processed to completion. Therefore, the neutral-prime condition is suitable as an indication of both target facilitation and inhibition when the SOA between prime and target is such that processing of the prime is complete. In this regard, it is important to note that the RT for the neutral-prime/word condition is roughly the same as the RTs for the unrelated word-word trials for both the low- and medium-proportion conditions, but falls between the overall RT of the unrelated word-word and related word-word conditions for the high-proportion condition. Regression equations obtained in Experiment 3 indicate that both costs and benefits increased as proportion increased. This further supports the present interpretation of an attention-driven, proportion-induced priming effect. Such an effect should include changes in both the amount of facilitation and the amount of inhibition as the proportion of related word-word pairs increases.

The interpretations with respect to the effect of SOA between prime and target on target processing, and the nature of the priming effects as a function of SOA, contain a potential inconsistency. It has been argued that target processing is slowed when the SOA between prime and target is brief-a finding also reported by others (Lorch, 1982; Schmidt, 1976). This implies that prime processing, or for that matter, target processing, is not automatic, because a common criterion of automaticity is that the automatic process does not, or should not, interfere with any other processing. At the same time, it is argued that the priming effect at a brief SOA is automatic. Thus, we appear to be arguing simultaneously for both automatic and attention-driven processes in the case of prime processing when the SOA between prime and target is brief. The simplest way of dealing with this apparent inconsistency is to distinguish between automaticity of prime processing and automatic effects of the prime once it has been processed to some level. These need not be correlated. Thus, although prime processing may involve some attentional component, or some structural limitation, it does not mean that the effect of the prime on the target cannot be automatic.

The point made in the preceding paragraph suggests that the term automatic should not be used globally in describing the processing of stimuli, no matter how familiar these stimuli may be to the observer. Rather, the processing of such stimuli as words, letters, etc., involves a number of aspects, some or most of which can be considered automatic and others of which involve capacity, or structural limitations requiring some form of attention. This conclusion is in accord with a recent review of attentional theory by Posner (1982), who makes a similar recommendation (p. 171).

In summary, the results of the present investigations verify the validity of the need to distinguish between two types of priming effects. These priming effects can be automatic in nature or strategic in nature. Furthermore, the effect of proportion of related trials in a priming procedure as reported by Tweedy (Tweedy \& Lapinski, 1981; Tweedy et al., 1977) appears to be mediated by attention-driven factors whose effects are absent when the SOA between prime and target is brief. The present research did not touch on the nature of the attention-driven priming effect other than to demonstrate that it can be induced by proportion of related trials and that its implementation is relatively slow. On the other hand, the nature of the automatic priming effect can be effectively explained by such models as the spreading-activation theory (Collins \& Loftus, 1975).

\section{REFERENCES}

Battig, W. F., \& Montague, W. E. Category norms for verbal items in 56 categories: A replication and extension of the Connecticut Category norms. Journal of Experimental Psychology Monograph, 1969, 80(3, Pt. 2).

Becker, C. A. Semantic context and word frequency effects in 
visual word recognition. Journal of Experimental Psychology: Human Perception and Performance, 1979, 5, 252-259.

Becker, C. A. Semantic context effects in visual word recognition: An analysis of semantic strategies. Memory \& Cognition, $1980,8,493-512$.

Clark, H. H. The language-as-fixed-effect fallacy: A critique of language statistics in psychological research. Journal of Verbal Learning and Verbal Behavior, 1973, 12, 335-359.

Collins, A. M., \& LofTus, E. F. A spreading-activation theory of semantic processing. Psychological Review, 1975, 82, 407-428.

Coltheart, $M$. Lexical access in simple reading tasks. In G. Underwood (Ed.), Strategies of information processing. London: Academic Press, 1978.

Fischler, I. Associative facilitation without expectancy in a lexical decision task. Journal of Experimental Psychology: Human Perception and Performance, 1977, 3, 18-26.

Fischler, I., \& Goodman, G. O. Latency of associative activation in memory. Journal of Experimental Psychology: Human Perception and Performance, 1978, 4, 455-470.

Foss, D. J., Cirilo, R. K., \& Blank, M. A. Semantic facilitation and lexical access during sentence processing: An investigation of individual differences. Memory \& Cognition, 1979, 7, 346-353.

LoRCH, R. F., Jr. Priming and search processes in semantic memory: A test of three models of spreading activation. Journal of Verbal Learning and Verbal Behavior, 1982, 21, 468-492.

Meyer, D. E., \& Schvaneveldt, R. W. Facilitation in recognizing pairs of words: Evidence of a dependence between retrieval operations. Journal of Experimental Psychology, 1971, 90, 227-234.

NeEly, J. H. Semantic priming and retrieval from lexical memory: Evidence for facilitatory and inhibitory processes. Memory \& Cognition, 1976, 4, 648-654.

NeELy, J. H. Semantic priming and retrieval from lexical memory: Roles of inhibitionless spreading activation and limitedcapacity attention. Journal of Experimental Psychology: General, 1977, 106, 226-254.

NEILL, W. T. Switching attention within and between categories: Evidence for intracategory inhibition. Memory \& Cognition, $1979,7,283-290$

Palermo, D. S., \& Jenkins, J. J. Word association norms:
Grade school through college. Minneapolis: University of Minnesota Press, 1964.

Posner, M. I. Cumulative development of attentional theory. American Psychologist, 1982, 37, 168-179.

Posner, M. I., \& Snyder, C. R. R. Attention and cognitive control. In R. L. Solso (Ed.), Information processing and cognition: The Loyola symposium. Hillsdale, N.J: Erlbaum, 1975. (a)

Posner, M. I., \& Snyder, C. R. R. Facilitation and inhibition in the processing of signals. In P. M. A. Rabbitt \& S. Dornic (Eds.), Attention and performance V. London: Academic Press, 1975. (b)

Schmidt, R. On the spread of semantic excitation. Psychological Research, 1976, 38, 333-353.

Schvaneveldt, R. W., \& McDonald, J. E. Semantic context and the encoding of words: Evidence for two modes of stimulus analysis. Journal of Experimental Psychology: Human Perception and Performance, 1981, 7, 673-687.

Stanovich, K. E., \& WEST, R. F. Mechanisms of sentence context effects in reading: Automatic activation and conscious attention. Memory \& Cognition, 1979, 7, 77-85.

Stanovich, K. E., \& West, R. F. The effect of sentence context on ongoing word recognition: Tests of a two-process theory. Journal of Experimental Psychology: Human Perception and Performance, 1981, 7, 658-672.

TweedY, J. R., \& LAPINSKI, R. H. Facilitating word recognition: Evidence for strategic and automatic factors. Quarterly Journal of Experimental Psychology, 1981, 33A, 51-59.

Tweedy, J. R., Lapinski, R. H., \& Schyaneveldt, R. W. Semantic-context effects on word recognition: Influence of varying the proportion of items presented in an appropriate context. Memory \& Cognition, 1977, 5, 84-89.

Whitten, W. B., Suter, W. N., \& Frank, M. L. Bidirectional synonym ratings of 464 noun pairs. Journal of Verbal Learning and Verbal Behavior, 1979, 18, 109-127.

Wike, E. L., \& Church, J. D. Comments on Clark's "The language-as-fixed-effect fallacy." Journal of Verbal Learning and Verbal Behavior, 1976, 15, 249-255.

(Manuscript received August 20, 1981; revision accepted for publication February 21, 1983.) 